\title{
Photobleaching with a Subnanosecond Laser Flash
}

\author{
Yifeng Yuan ${ }^{1}$ and Daniel Axelrod ${ }^{1-3}$
}

Received August 4, 1993; revised January 4, 1994; accepted January 6, 1994

\begin{abstract}
In standard fluorescence recovery after photobleaching (FRAP) applications for measuring lateral diffusion rates and adsorption/desorption kinetics of fluorescent molecules at biological or model membranes, irreversible bleaching is induced by a bright excitation flash of at least millisecond time scale. It has been presumed that the bleaching event is of a low probability and the significant bleached population that develops during the flash results from each molecule undergoing thousands of excitation/deexcitation cycles before a bleaching event occurs. In some FRAP experiments, notably polarized FRAP (PFRAP) for measuring molecular rotational diffusion rates, it is desirable to use much shorter (subnanosecond) bleaching pulses. However, subnanosecond pulses are shorter than the fluorescence lifetime, so that any fluorophore will experience at most only one visit to the excited state during the bleaching pulse. If bleaching occurs only by the same processes as in slower FRAP experiments, one would thereby expect only minimal bleaching regardless of the bleach intensity. Moreover, the ability of fast polarized pulses to imprint an anisotropic orientational pattern in the postbleach unbleached fluorophore, an ability essential for PFRAP, is not at all guaranteed, particularly if two-photon processes are involved in high-intensity short bleach pulses. In this study, bleaching depths are measured as a function of subnanosecond pulse intensity on a small labeled protein covalently immobilized on fused silica. We show that bright subnanosecond laser flashes do indeed produce significant bleaching, that both two photon effects and reversible bleaching are involved, and that polarized bleaching does produce an anisotropic orientational pattern of unbleached fluorophore. We also postulate a theoretical molecular state model which semiquantitatively accounts for the experimentally observed dependence of reversible bleaching on bleaching pulse intensity.
\end{abstract}

KEY WORDS: Polarization; two-photon effects; fluorescence; pulsed laser; rotational.

\section{INTRODUCTION}

Fluorescence recovery after photobleaching (FRAP) techniques [1] are used routinely to measure lateral diffusion rates and adsorption/desorption kinetics of fluorescent molecules at biological or model membranes. In these techniques, irreversible bleaching is induced by

' Biophysics Research Division, University of Michigan, Ann Arbor, Michigan 48109.

2 Department of Physics, University of Michigan, Ann Arbor, Michigan 48109.

${ }^{3}$ To whom correspondence should be addressed at Biophysics Research Division, University of Michigan, Ann Arbor, Michigan 48109. a bright excitation flash of at least millisecond time scale. The bleaching event itself may destroy the excited fluorophore itself or it may destroy a ground-state fluorophore nearby. But either way, it has been presumed that the bleaching event is of a low probability (e.g., once in several thousand excitations) and the significant bleached population that develops during the flash results from each molecule undergoing thousands of excitation/deexcitation cycles before a bleaching event occurs.

In recent years, polarized FRAP (PFRAP) [2] and a related variant called polarized fluorescence depletion (PFD) [3] have been developed to study the rotational 
diffusion rates of proteins on cell surface, by use of polarized bleach pulses and polarized probe excitation and emission detection through a microscope. Because of the very short (nanosecond) time scales possible in such motion, it is desirable to use extremely short (subnanosecond) bleaching pulses. The efficacy of using such short pulses has not been examined heretofore, and several considerations suggest that the bleaching phenomena familiar at longer times are not relevant at shorter times. (a) Subnanosecond pulses are shorter than the fluorescence lifetime, so that most fluorophores will experience at most only one visit to the excited state, rather than thousands, before the bleaching pulse is over. If bleaching occurs only by the same processes as in slower FRAP experiments, one would thereby expect only minimal bleaching regardless of the bleach intensity. (b) If significant bleaching does occur, it must involve mechanisms not seen with longer and dimmer bleach intensities, such as reversible bleaching (in which the bleached fluorophores recover their capability for fluorescence excitation over time) or two-photon dependent bleaching. (c) The ability of fast polarized pulses to imprint an anisotropic orientational pattern in the postbleach unbleached fluorophore, an ability essential for PFRAP, is not at all guaranteed, particularly if two-photon processes are involved.

We show here on a suitable experimental system the surprising results that subnanosecond laser flashes do indeed produce significant bleaching, that both twophoton effects and reversible bleaching are involved, and that polarized bleaching does produce an anisotropic orientational pattern of unbleached fluorophore. Bleaching depths are presented as a function of pulse intensity. We also postulate a theoretical molecular state model which semiquantitatively accounts for the experimentally observed dependence of reversible and irreversible bleaching on bleaching pulse intensity.

\section{EXPERIMENTAL}

We examined the bleaching depth and postbleach recovery of tetramethylrhodamine labeled $\alpha$-bungarotoxin (R- $\alpha B G T$ ) [4] in response to a single polarized 0.5 -ns-duration pulsed laser flash. This particular labeled protein was chosen because it is the marker of acetylcholine receptors in PFRAP experiments on developing muscle cells currently proceeding in our lab. The fluorescence lifetime of $\mathrm{R}-\alpha \mathrm{BGT}$ was not directly measured. However, other indirect evidence suggests that it is significantly longer than $0.5 \mathrm{~ns}$. The lifetime of a similar fluorophore, rhodamine B in water, is about 2-
3 ns $[5,6]$. Rhodamine $B$ and tetramethylrhodamine have comparable absorption spectra and fluorescence brightness. Upon monosubstituted conjugation to $\alpha$-bungarotoxin, tetramethylrhodamine suffers only a slight decrease in fluorescence quantum efficiency, from 0.35 to 0.28 [4]. Therefore, we surmise that the fluorescence lifetime of $R-\alpha B G T$ is in the 2- to 3-ns range.

In all of the experiments here, the protein is immobilized on a solid substrate so that orientational anisotropy of photobleaching by polarized light could be examined without the randomizing action of rotational diffusion. This section discusses sample preparation, the optics of the PFRAP system, and data acquisition.

\section{Sample Preparation}

Rhodamine monolabeled $\alpha$-bungarotoxin [4] was covalently attached via an organosilane to $1 \times 1$ in. fused silica microscope slides (Heraeus Amersil Inc., Sayreville, NJ) using a technique adapted by Fulbright and Axelrod [7] for coupling protein to fused silica substrates. (Use of fused silica avoids the strong transient luminescence of ordinary glass within the first millisecond after a bright flash of visible light). After covalent attachment of the R- $\alpha B G T$, the slide is rinsed once in $\mathrm{H}_{2} \mathrm{O}$, twice in $4 \mathrm{MNH}_{4} \mathrm{Cl}$, and then five times in $\mathrm{H}_{2} \mathrm{O}$ to ensure that the substrate-associated protein is irreversibly bound. This finished slide was stored under vacuum in a freezer if not used immediately. The PFRAP experiments were performed on this sample immersed in Hank's balanced salt solution (HBSS). All of the samples were in equilibrium with air.

\section{Optical System for Bleaching}

The optical system is a much speeded-up version [8] of the PFRAP apparatus previously described [2,911 , now capable of bleaching at 0.5 -ns pulse duration and recording recoveries down to 10-ns sample times. The bleach is induced by a 514-nm flash from an $\mathrm{N}_{2} /$ dye laser (Laser Photonics). The postbleach recovery is excited ("probed") by a polarized CW argon laser beam set at $514 \mathrm{~nm}$. The dimensions of the pulsed laser bleach at the sample are quite large for a FRAP experiment, about a $2-\mu \mathrm{m}$ radius, and the radius of the approximately Gaussian probe beam is $-0.5 \mu \mathrm{m}$. The details are described in the legend to Fig. 1.

Fluorescence intensity was monitored by an avalanche photodiode unit called a single-photon counting module (SPCM) (EG\&G), mounted atop an inverted epifluorescence microscope (Leitz Diavert, with a $40 \times$, NA 0.75, water immersion objective). The SPCM output 


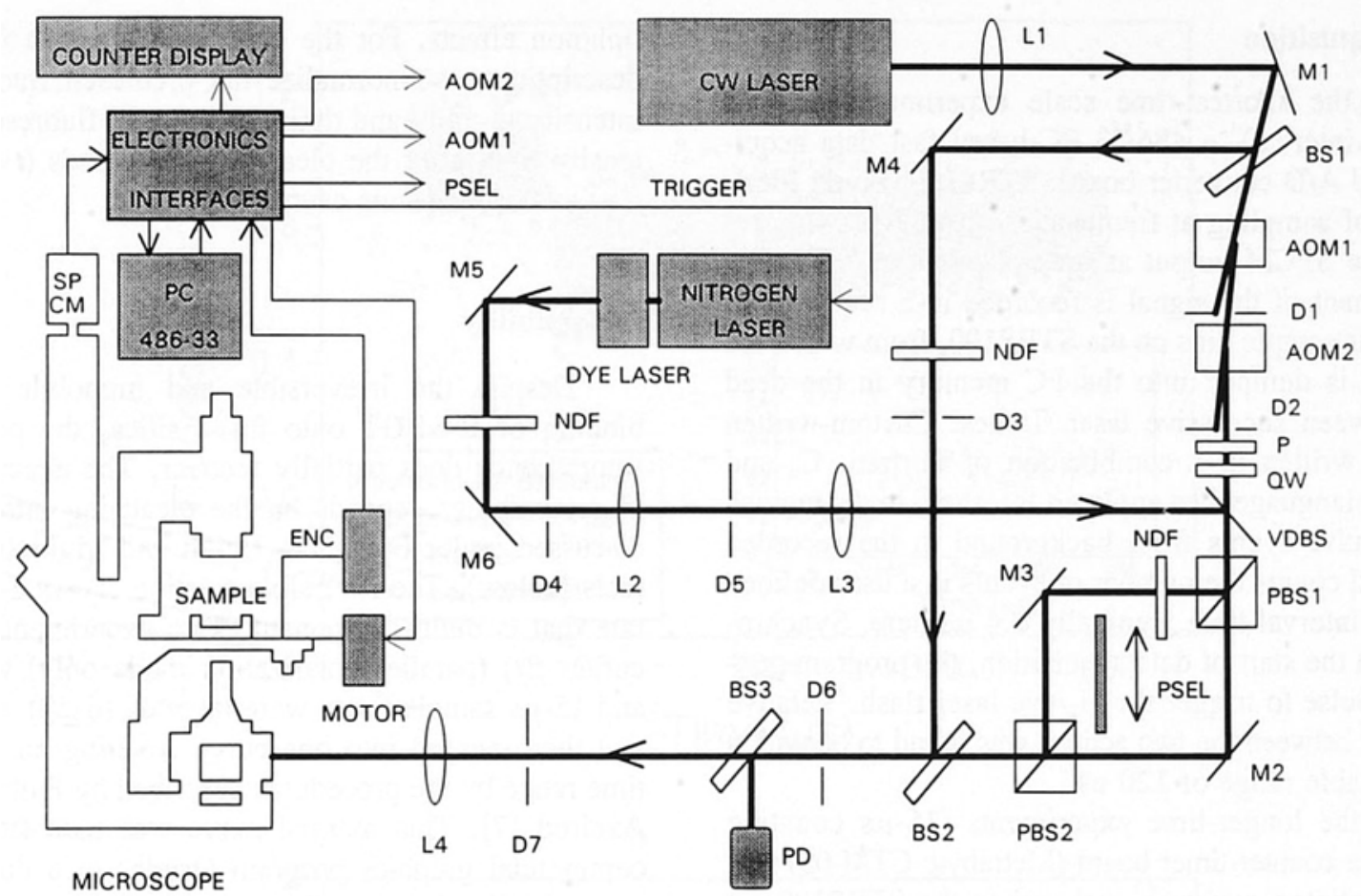

Fig. 1. This system interchangeably uses either a CW argon laser (Coherent, Inc.; 15-W Innova 20, output of 4-6 W at $514 \mathrm{~nm}$ ) or a nitrogen laser-pumped dye laser (Laser Photonics, Inc.; output of $\sim 250 \mu \mathrm{J} /$ pulse tuned at $514 \mathrm{~nm}$ ) as the bleaching beam source. The CW laser is always the source of the probe beam.

For the case of $\mathrm{CW}$ laser bleaching, the horizontally polarized laser beam passes through a long-focal length lens (L1), through two successive acousto-optic modulators (AOM 1 and AOM 2) activated by the same signal, to reach a focus at a diaphragm (D2) that transmits only the firstorder diffraction beam from the modulators. (Two modulators are used in series to ensure a very high, 1,000,000:1, contrast ratio between the bright vs very dim throughput with the AOM's activated vs nonactivated state.) The beam continues through a horizontal polarizer prism ( $P$ ) and a quarter wavcplatc (QW) to produce circular polarization.

For the case of pulsed laser bleaching, an alternate path is used up to this same point. The unpolarized pulse beam is focused by lens $\mathrm{L} 2 \mathrm{Lthrough}$ a circular diaphragm (D5), recollimated by lens L3, and joined to the subsequent path of the bleach beam by a variable-density beam splitter (VDBS).

The bleach beam is then split by a polarizing cube (PBS1): The horizontally polarized component passes straight through and the vertically polarized component is deflected $90^{\circ}$; the relative intensity of these two polarizations is adjusted by a neutral density filter (NDF). During the dead time between each bleach/rccovery cycle, either one of these two orthogonal polarized bleaching beams is alternately selected for the bleach of the next cycle by blocking the other polarization with a solenoid-driven flag (PSEL) under computer control.

The probe beam is always gencrated by the CW laser, regardless of the source of the bleach beam, and it always has a fixed polarization (horizontal). Before the CW laser beam encounters the AOMs, the probe beam path is created by reflection from the surface of a $45^{\circ}$ optical flat beam splitter (BS1). The probe path is then recombined with the bleach path at BS2 after the latter has passed through the polarization-switching system.

Both the bleach and the probe beams pass through a simple converging lens $\mathrm{L} 4$ which focuses them onto the field diaphragm plane of an epifluorescence microscope (Leitz Diavert). The beams reflect a dichroic mirror up through the objective (Zeiss $40 \times, N A=0.75$, water immersion) and onto the sample. The emitted fluorescence, captured by the same objective and transmitted through the dichroic mirror, passes through a film polarizer, which is always oriented to transmit parallel to the polarization direction of the horizontally polarized bleach beam.

SPCM, single-photon counting module; ENC, linear encoders for tracking the stage motion; PD, photodiode for monitoring probe beam intensity.

consists of TTL-sized photon counts of 200-ns duration, thereby limiting its maximum usable count rate to about $500,000 / \mathrm{s}$ and the minimum sample time to $0.4 \mu \mathrm{s}$. But the SPCM's major advantages over standard photomultiplier tubes in this application are its insensitivity to electrical transients triggered by the pulse laser flash and its resilience against bright flashes of light even without electronic or optical gating.

Bleaching light intensity is varied by inserting appropriate neutral density filters into the bleaching beam path. The highest value of the bleaching intensity is approximately $10^{27}$ photons $/ \mathrm{cm}^{2}$-s on the sample. 


\section{Date Acquisition}

For the shortest-time scale experiments $(0.4-\mu \mathrm{s}$ counting interval), a 486/33 PC-based fast data acquisition and A/D converter board (STR8100, Sonix Inc.), capable of sampling at frequencies up to $100 \mathrm{Mhz}$, receives the SPCM output as an analog input. The time development of the signal is recorded in a sequence of $64 \mathrm{~K} 10$-ns sample bins on the STR8100, from which the sequence is dumped into the PC memory in the dead time between successive laser flashes. Custom-written software written in a combination of Fortran, C, and assembly language then applies a threshold to distinguish photon pulse events from background in the recorded signal and counts the number of events in a user-defined counting interval time, generally $0.4 \mu$ s here. Synchronous with the start of data acquisition, the program generates a pulse to trigger the $\mathrm{N}_{2}$ /dye laser flash. Relative time jitter between the two actions was found to be within an acceptable range of 120 ns.

For the longer-time experiments $(15-\mu \mathrm{s}$ counting interval), a counter-timer board (Metrabyte CTM-05) was used for photon counting, rather than the STR 8100 , as described previously $[2,9-11]$.

In PFRAP, signal averaging over thousands of flash/ recovery cycles is necessary. Each flash is performed in a new area of the sample, with an automatic microscope stage motion driven by stepper motors (Compumotor PC23 system) and directed by the same custom program. The $5-\mu \mathrm{m}$ step size is just large enough to ensure that adjacent bleached regions do not overlap.

To distinguish rotational diffusion (which gives recoveries that depend on the polarization of the bleaching flash) from reversible photobleaching (which gives polarization-independent recoveries), successive bleaches are made to alternate between two orthogonal polarizations: One is parallel and the other is perpendicular to the (constant) probe polarization [2]. The bleach polarization is determined by a solenoid-operated beam blocker, the software programmed position of which alternates in the dead time between each flash/recovery cycles.

\section{RESULTS}

Typical fluorescence recovery curves after subnanosecond bleaching are shown in Figs. $2 a$ and $b$ for R$\alpha B G T$ covalently attached to fused silica, for the two sample times of data acquisition, 0.4 and $20 \mu \mathrm{s}$, respectively. We discuss here several features of such curves: (a) reversibility of the bleach, (b) bleaching polarization anisotropy, and (c) bleaching depth and mul- tiphoton effects. For the convenience in the following descriptions, we normalize the prebleach fluorescence intensity to unity and define $f(0)$ as the fluorescence intensity right after the bleaching pulse ends $(t=0)$, and $f(\infty)$ as the long-time asymptote.

\section{Reversibility}

Despite the irreversible and immobile covalent binding of R- $\alpha$ BGT onto fused silica, the postbleach fluorescence does partially recover. The exact fraction of reversibility depends on the bleaching intensity, as discussed under Bleaching Depth and Multiphoton Effects (below). The reversible fraction shows a recovery rate that is multi-component. Two experimental FRAP curves $f(t)$ (parallel polarization mode only) with 0.4and $15-\mu$ s sample times were inverted to $g(t) \equiv 1-f(t)$ and then merged into one curve covering an extended time range by the procedures described by Fulbright and Axelrod [7]. This merged curve was then fit (by the commercial graphics program Origin) to a double exponential (for the reversible part) plus a constant (for the irreversible part). The characteristic times (and relative fractions) of the reversible part were relatively insensitive to bleaching intensity; they are $20 \mu \mathrm{s}(57 \%)$ and $2.3 \mathrm{~ms}(43 \%)$.

\section{Bleaching Anisotropy}

If the pulsed $\mathrm{N}_{2} /$ dye laser is to be usable for PFRAP, we require that the parallel-mode bleaching depths at $t=0$ must be greater than those obtained in the perpendicular mode. Indeed, relaxation of this difference due to the rotational motion of a fluorophore is the essence of the PFRAP technique. Figure 2 shows that significant $t=0$ bleaching anisotropy is indeed generated by pulsed laser bleaching. The bleaching anisotropy $r_{b}$ is defined as

$$
r_{\mathrm{b}}(0) \equiv\left(\Delta f_{\square}-\Delta f_{\perp}\right) /\left(\Delta f_{\|}+2 \Delta f_{\perp}\right)
$$

where $\Delta f_{\|, \perp}-f_{\|, \perp}(0)$ is the amount of bleached fluorescence (normalized to the prebleach value) in the parallel and perpendicular polarization modes at $t=0$, respectively. The larger $r_{b}(0)$, the more feasible is a PFRAP experiment. A typical CW laser bleaching flash on $\mathrm{R}$ $\alpha B G T$, with a duration of at least tens of microseconds, gives $r_{\mathrm{b}}(0) \approx 0.1$. But the 0.5 -ns pulsed laser gives an even larger $r_{b}(0) \approx 0.2$. With both types of laser bleaching, there is a moderate decrease in bleaching anisotropy with an increasing amount of bleaching. 

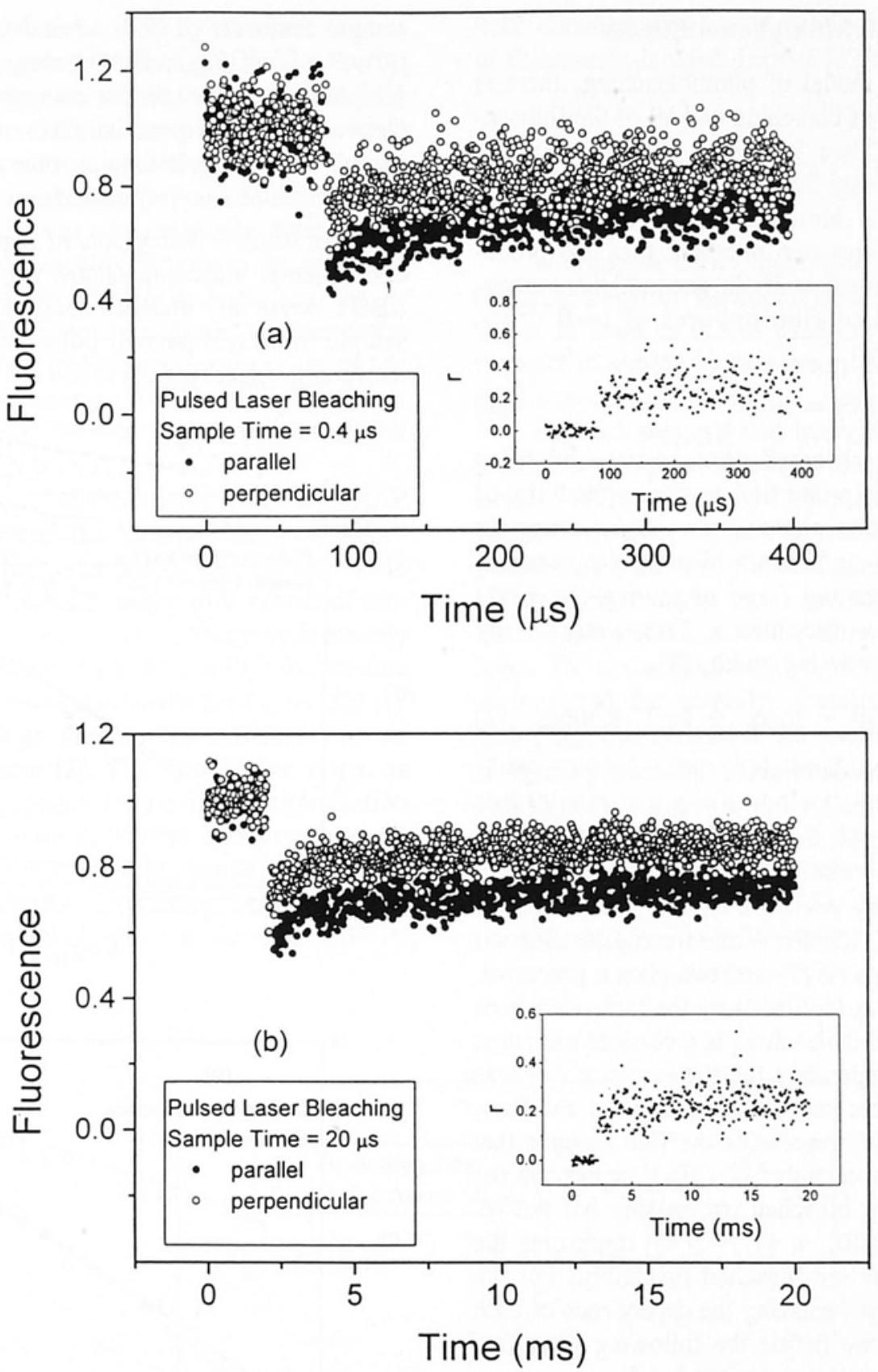

Fig. 2. Typical PFRAP curves of subnanosecond bleaching of immobilized R- $\alpha$ BGT for (a) $0.4-\mu s$ and (b) 20- $\mu$ s sample times. PFRAP curves for the bleaching polarization parallel (filled circles) and perpendicular (open circles) relative to the emission polarizer and probe beam polarization are shown. The bleach intensities were not exactly the same in $a$ and $b$. Note that both the parallel and the perpendicular curves increase monotonically. This indicates that a polarization-independent process contributes to the time course of fluorescence recovery [2]. Since the sample is immobile, that recovery process is likely to involve molecular-state transitions rather than diffusion. The inset in each graph shows the corresponding bleaching anisotropy $r_{\mathrm{b}}(t)$. The fact that $r_{\mathrm{b}}(t)$ does not decay to zero indicates that the R- $\alpha \mathrm{BGT}$ is rotationally immobile on the time scales shown. 


\section{Bleaching Depth and Multiphoton Effects}

In the simplest model of photobleaching, there is only one mechanism of bleaching and all of the fluorophores are identical. Then for single-photon bleaching, the probability rate of any fluorophore becoming bleached is proportional to the bleach intensity $I$. But if each bleaching event requires two photons, then the probability rate is proportional to $I^{2}$. In general, the concentration of unbleached fluorophores at $t=0$ drops exponentially such that

$$
f(0)=e^{-\alpha_{n} T T^{n}}
$$

where $n=1$ or 2 absorbed photons required for a bleaching event, $\alpha_{n}$ is proportional to the probability of bleaching per excitation photon (or pair) for a one (or two-)-photon process, and $T$ is the bleach pulse duration. By experimentally varying $I$ and measuring the corresponding $f(0)$, we can determine $n$. This is most easily done by twice taking the log of Eq. (2).

$$
B \equiv \log \{-\log [f(0)]\}=\log \alpha_{n}+\log T+n \log I
$$

If the experimentally determined left-side quantity is plotted against $\log I$, the straight-line graph should have a slope of $n$. (Of course, the quantitative position along either axis is arbitrary and depends on the units chosen for $\alpha_{n}, T$, and $I$.) Any deviation from a straight line or a nonintegral slope indicates a more complex process, possibly involving both single- and two-photon processes.

The situation is complicated by the fact, seen from Fig. 2, that some of the bleaching is reversible and some is irreversible. $f(0)$ represents the fluorescence that was not bleached by either mechanism: It is the total unbleached fraction. $f(\infty)$ represents the fluorescence that was not bleached irreversibly. $[1-f(\infty)]$ represents the fluorescence that was bleached irreversibly but not reversibly. Therefore, $f(0)+[1-f(\infty)]$ represents the fluorescence that was not bleached reversibly. For the purpose of graphically displaying the dependence of each mechanism upon $I$, we define the following bleaching depth ordinates to be plotted against $\log I$ :

$$
\begin{aligned}
B_{\mathrm{tot}} & \equiv \log \{-\log [f(0)]\} \\
B_{\text {ir }} & \equiv \log \{-\log [f(\infty)]\} \\
B_{\text {rev }} & \equiv \log \{-\log [f(0)-f(\infty)+1]\}
\end{aligned}
$$

Note that the experimental estimate of the fluorescence $f(0)$ at $t=0$ is actually the fluorescence measured in the first postbleach sample bin. In the experiments (and in the theoretical model discussed below), we use sample times $\Delta t$ of both 15 and $0.4 \mu$ s. In principle, $f(0), B_{\text {rev, }}$ and $B_{\text {tot }}$, will then depend somewhat on the sample time, since the fast component of the reversible recovery has a characteristic time $(20 \mu \mathrm{s})$ that is comparable to the sample time in some experiments $(15 \mu \mathrm{s})$. $B_{\text {irt }}$ is based on the $f(\infty)$ value from the $15-\mu$ s runs only.

The discrete data points in Figs. 3a and $b$ show the experimental bleaching depths $B_{\mathrm{tot}, \mathrm{ir}, \mathrm{rev}}$ vs $\log I$ for R$\alpha B G T$ covalently attached to fused silica, for the two sample times and parallel polarization mode only. The irreversible bleaching curve has the steepest slope, about
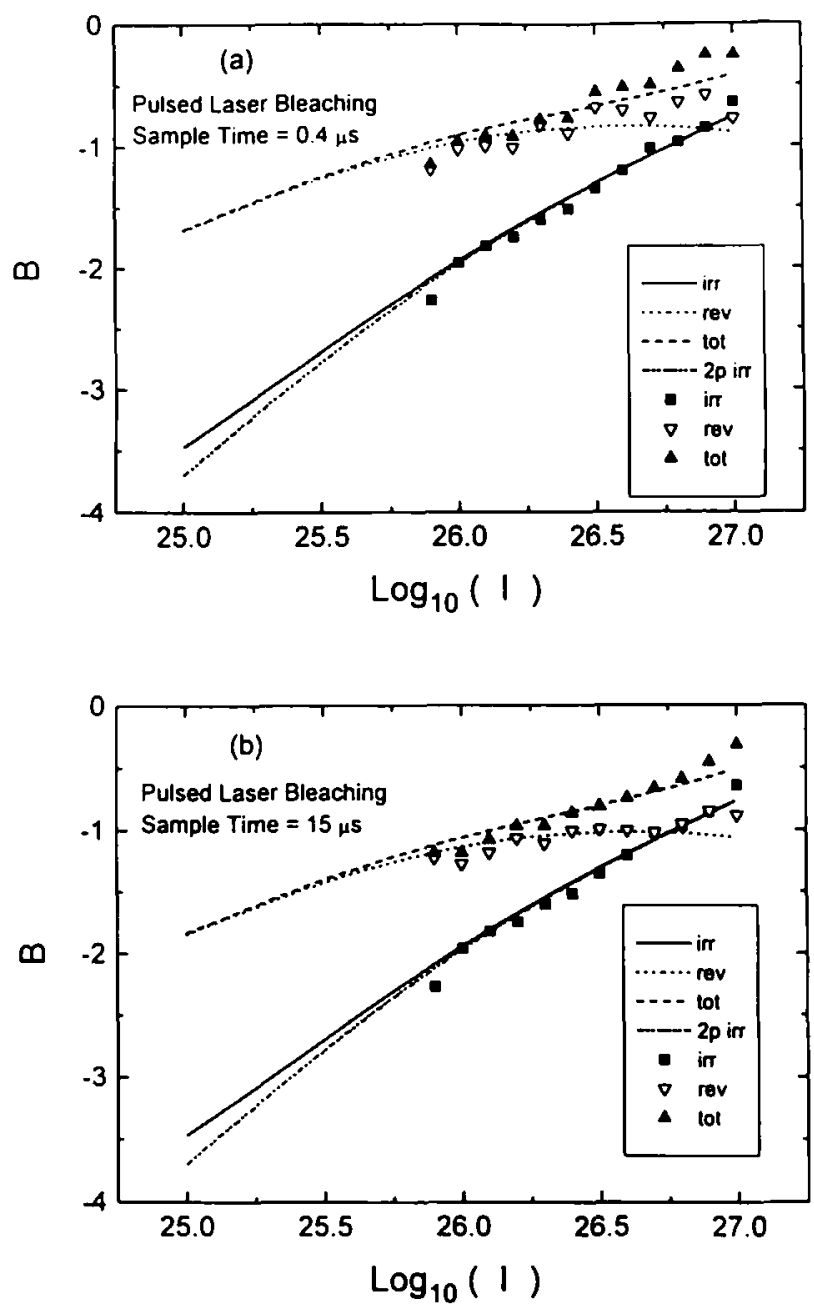

Fig. 3. Bleaching depth parameter $B$ vs $\log I$ for subnanosecond bleaching, for (a) 0.4- $\mu$ s and (b) 15- $\mu$ s sample times. Theoretical results for $B_{\text {ir, rev.tot }}$ are shown with solid, dotted, and dashed lines, respectively. Corresponding experimental results are shown as filled squares, inverted open triangles, and filled triangles, respectively. The " $2 p$ irr" theoretical curve shows how the irreversible curve would appear if it were generated exclusively from the hypothesized two-photon process. 
$1.38 \pm 0.05$, and the slope is fairly constant over at least one order of magnitude variation in $I$. The reversible bleaching curve has the smallest slope, about 0.64 \pm 0.09 , in the middle of the intensity range, and it tends to flatten to near-zero at the highest intensities. The total bleaching curve slope is intermediate, about $0.68 \pm 0.09$, and is fairly constant. Interpreted physically, these curves show that reversible bleaching dominates at low light end. As the bleaching light intensity increases, the irreversibly bleached fluorophore population increases faster than the reversibly bleached fluorophore population. At the high-intensity end, irreversible bleaching begins to dominate. The smooth lines in Fig. 3 are theoretical models, discussed below.

In contrast to the above experiments, standard FRAP experiments described in the literature have used $C W$ lasers for both bleaching and probe, and the time scale is much longer, usually milliseconds to hundreds of seconds. At these long times, reversible recovery is already complete and all that remains is the familiar irreversible bleaching. Figure 4 shows the results for $B_{\text {irr }}$ vs $\log I$ for a CW bleach of $15-\mu$ s duration as modulated by an acousto-optic modulator [2]. The straight-line slope, as fit by a nonlinear regression least-squares minimization procedure (included in the GraphPad Inplot commercial graphing program), is $0.96 \pm 0.05$, which is very close to the 1.0 expected from a simple single-photon mechanism. It is also consistent with the slope of $1.08 \pm$
0.12 obtained for slow bleaching on aqueous solutions of fluorescein-labeled dextran [12].

\section{THEORY}

We propose here a theoretical model for explaining the mechanism of fluorophore photobleaching by laser pulses as short as 0.5 ns used in our experiments. For the sake of simplicity, we ignore bleaching polarization in this model.

Figure 3 suggests that irreversible bleaching might involve a two-photon process because the $B_{\text {irr }}$ vs $\log I$ slope $(=1.38 \pm 0.05)$ is significantly larger than unity. In this model, we assume that it is a resonant two-photon process; i.e., the first photon excites the molecule to the lowest excited singlet state, and while it is in that state, the second photon excites the molecule to an even higher level. The consequent high state of excitation is then the springboard for virtually certain irreversible photobleaching. Such a model can account for why the slope of $B_{\text {iтr }}$ vs $\log I$ is less than the 2.0 value that one might expect of a two-photon process. In the limit of a low light level, the rate of each of the transitions is proportional to $I$, so the rate of the whole two-transition process is proportional to $P^{2}$. But if the light is bright enough, the intermediate state can approach saturation and the

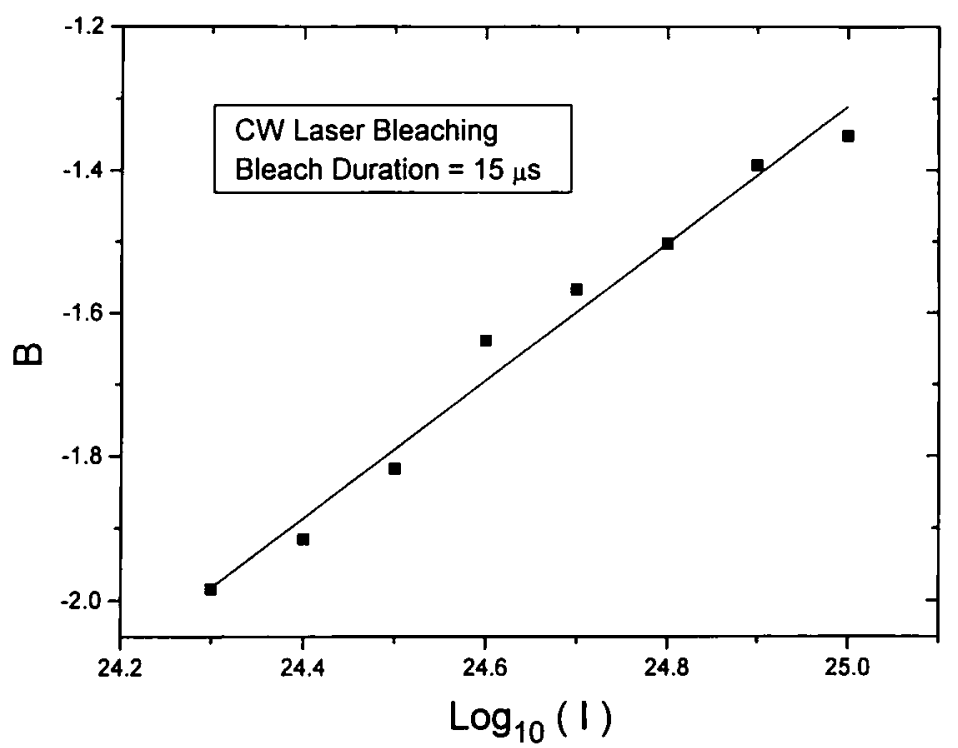

Fig. 4. Blcaching depth parameter $B$ vs $\log l$ for $15-\mu \mathrm{s}$-duration bleaching with an AOM-modulated CW laser. Experimental results are discrete points; the solid line is a linear best fit. 
whole process then approaches only first-order proportionality to $I$.

Figure 3 also suggests that the model should involve a reversible photobleaching pathway. Here we assume that this is trapping in a long-lived triplet state. We observed that the characteristic time of reversible recovery was of the order of 10 to $100 \mu \mathrm{s}$, which is in the range of triplet-state lifetimes. Previous experimental observations have shown that oxygen (which quenches triplet states) decreases both the efficiency and the recovery time of reversible bleaching [10]. Reversible bleaching could also be due to other effects such as reversible photoinduction of a nonfluorescent dimer [13].

Thus we propose a simple model consisting of the ground state $(G)$, the first excited singlet state (S), and the lowest triplet state $(T)$, along with transition rates among them as shown in Fig. 5. The model also contains routes for irreversible photobleaching, represented by transitions out of the system from the excited singlet state (induced by a second photon) and from the triplet state (occurring spontaneously). In actuality, irreversible bleaching may involve the excited fluorophore donating its energy to molecular oxygen, which then enters its own highly reactive excited singlet state. The excited singlet oxygen then destroys molecules in the vicinity [14], perhaps even neighboring fluorophores in the ground state. It is reasonable to hypothesize that the second pho-

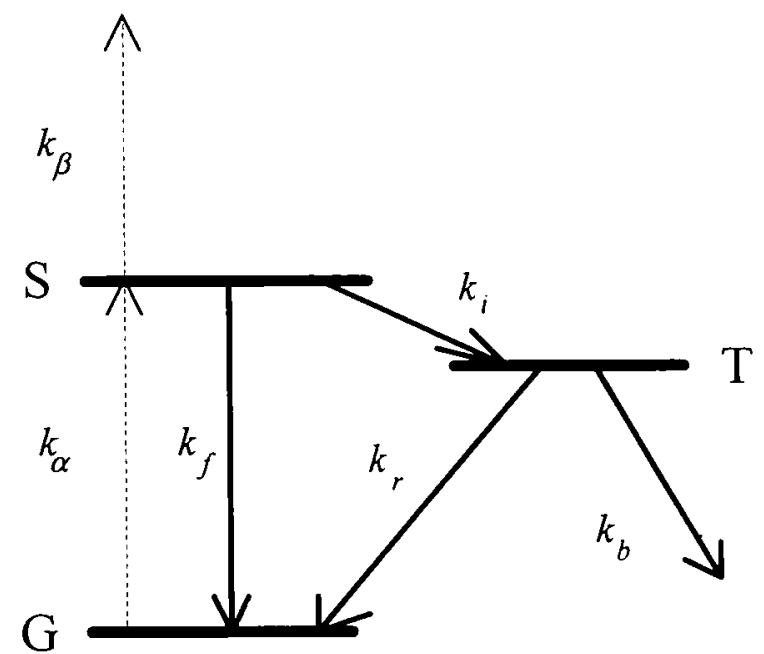

Fig. 5. Molecular-state schematic diagram of our kinetic model for subnanosecond bleaching. $\mathrm{G}$, ground state; $\mathrm{S}$, lowest excited-singlet state; T, triplet state. Kinetic rate $k$ subscripts: $\alpha$, single-photon absorption; $\beta$, second-photon absorption, presumed always to Icad to irreversible bleaching; $f$, fluorescence and radiationless decays; $i$, intersystem crossing, presumed always to lead to cither reversible bleaching recovery $r$ or to single-photon irteversible bleaching $b$. ton, absorbed by molecules already in the S state, might lead to irreversible bleaching: rhodamine dye emits electrons when exposed to light over the wavelength range 180 to $270 \mathrm{~nm}$ [15].

The transition kinetic rates are as follows: $k_{\alpha, \beta} I$ are the light absorption kinetic rates up from the ground state and singlet excited state, respectively, expressed as products of the incident light intensity and a factor proportional to the absorption cross section; $k_{f}$ is the $\mathrm{S} \rightarrow \mathrm{G}$ decay kinetic rate, which includes both fluorescence and radiationless decay working in parallel; and $k_{\mathrm{i}}$ is the intersystem crossing rate. A fluorophore in the triplet state either decays to the ground state with kinetic rate $k_{\mathrm{r}}$ or becomes irreversibly bleached with kinetic rate $k_{\mathrm{b}}$.

All reversible bleaching (presumed to be single photon) follows the path characterized by $k_{\mathrm{r}}$ and singlephoton irreversible bleaching (normally observed as the predominant mode in $\mathrm{CW}$ laser bleaching experiments) follows the path characterized by $k_{\mathrm{b}}$. The two-photon irreversible bleaching process follows the path characterized by the sequence of $k_{\alpha}$ followed by $k_{\beta}$. The timedependent populations of the ground, singlet, and triplet states are denoted $N_{\mathrm{G}}, N_{\mathrm{S}}$, and $N_{\mathrm{T}}$, respectively. Irreversible bleaching, whether of the one- or two-photon variety, can be viewed as depositing fluorophores into a fourth state from which they never return, with a population $N_{\mathrm{B}}$. Counting all these four "states," the total number of fluorophores $N$ is a constant. The populations are described by the following kinetic equations:

$$
\begin{aligned}
\frac{d N_{G}}{d t} & =-k_{\alpha} N_{G} I(t)+k_{f} N_{\mathrm{S}}+k_{\mathrm{r}} N_{\mathrm{T}} \\
\frac{d N_{S}}{d t} & =k_{\alpha} N_{G} I(t)-\left[k_{\beta} I(t)+k_{i}+k_{f}\right] N_{\mathrm{S}} \\
\frac{d N_{T}}{d t} & =k_{i} N_{S}-\left(k_{r}+k_{b}\right) N_{T} \\
\frac{d N_{B}}{d t} & =k_{\beta} N_{S} I(t)+k_{i} N_{T} \\
N & =N_{G}+N_{S}+N_{T}+N_{B}
\end{aligned}
$$

The incident intensity $I(t)$ is given by

$$
I(t)= \begin{cases}I & \left(-t_{B}<t<0\right) \\ 0 & (0<t<\infty)\end{cases}
$$

where $t_{\mathrm{B}}$ is the bleach duration. Actually, $I(t)$ is slightly larger than zero at all times after the bleach (during the probe phase of FRAP), but we assume that no further bleaching occurs and then $I(t)$ is bright enough only to excite fluorescence proportional to the population $N_{\mathrm{G}}$.

The above system of equations can be solved ana- 
lytically by standard methods for the normalized populations $n_{\mathrm{G}, \mathrm{S}, \mathrm{T}, \mathrm{B}}(t) \equiv N_{\mathrm{G}, \mathrm{S}, \mathrm{T}, \mathrm{B}}(t) / N$. But the explicit solutions are complicated looking, so we show graphs of $B_{\text {tot,irr, rev }}$ vs $\log I$ as the smooth curves in Fig. 3, superimposed on the experimental data. In this theoretical case,

$$
\begin{aligned}
B_{\mathrm{tot}} & \equiv \log \left\{-\log \left[n_{G}\left(t_{B}\right)\right]\right\} \\
B_{\mathrm{irr}} & \equiv \log \left\{-\log \left[n_{G}(\infty)\right]\right\} \\
B_{\mathrm{rev}} & \equiv \log \left\{-\log \left[n_{G}\left(t_{B}\right)-n_{G}(\infty)+1\right]\right\}
\end{aligned}
$$

The experimentally measured fluorescence intensity $f(t)$ at any time $t$ is not instantaneous but, rather, an average over the duration of one sample time $\Delta t$. Correspondingly, $n_{\mathrm{G}}\left(t_{\mathrm{B}}\right)$ is calculated as the average fluorophore population in the time interval $\left(t_{\mathrm{B}}, t_{\mathrm{B}}+\Delta t\right)$. The theoretical curves in Figs. 3a and $\mathrm{b}$ use $\Delta t=0.4$ and $15 \mu \mathrm{s}$ respectively, corresponding to the experimental data.

The experimental recovery curves showed there have at least two components to the reversible recovery: a fast one, of a characteristic time $20 \mu \mathrm{s}$; and a slow one, of the order of $2.3 \mathrm{~ms}$. One possibility is that the environment of the rhodamine groups is heterogeneous. For the purposes of this theoretical calculation, we thereby assumed that $57 \%$ of the rhodamine had a triplet lifetime of $20 \mu \mathrm{s}\left(k_{\mathrm{r}}=5 \times 10^{4} \mathrm{~s}^{-1}\right)$, and the other $43 \%$ had a lifetime of $2.3 \mathrm{~ms}\left(k_{\mathrm{r}}=440 \mathrm{~s}^{-1}\right)$, and we averaged the theoretical results arising from the two rates.

For a certain set of kinetic parameters $k_{\mathrm{i}, \mathrm{r}, \mathrm{f}, \mathrm{b}, \alpha, \beta}$ (see below), the theoretical model curves can be made to agree semiquantitatively with the experimental data. This agreement is nontrivial because the theoretical and experimental curves both exhibit the following similarities in their relative positions and shapes.

(a) At the low-intensity end, almost all of the bleaching is reversible. The very high probability of a fluorophore following this pathway accounts for why measurable bleaching can be accomplished even with a 0.5 -ns duration laser pulse. Because of its rapidity, the reversibility is not normally seen at the longer time scales more conventionally used in FRAP.

(b) At the high-intensity end, the reversible bleaching depth becomes less important and even declines somewhat with increasing $I$ as it loses the competition for excited singlet fluorophores with irreversible two-photon bleaching.

(c) The irreversible bleaching component has a middle-intensity range in which the dependence on $I$ is faster than linear, but it is never quite as fast as $I^{2}$. At either end of the intensity scale, the dependence on $I$ is even slower. At middle to high intensities, the excited singlet state begins to saturate. At low intensities, the singlephoton irreversible bleaching rate $k_{\mathrm{b}}$ dominates. This effect becomes clear if we calculate the curves assuming that $k_{\mathrm{b}}=0$, thereby leaving only two-photon bleaching and an $I^{2}$ dependence; this alternative is depicted as the " $2 p$ irr" (twophoton irreversible) curve in Figs. $3 a$ and $b$.

(d) The plot of total of irreversible and reversible bleaching depths $B_{\text {tot }}$ vs $I$ never achieves a slope larger than unity at any intensity range, and the slope even decreases at the high end despite the dominance of two-photon bleaching there.

(e) The apparent reversible bleaching for the $15-\mu \mathrm{s}$ sample time (Fig. 3b) is shallower than that for the 0.4- $\mu$ s sample time (Fig. 3a) for both theory and experiment. This is expected since some of the reversible recovery occurs on the time scale of only $20 \mu \mathrm{s}$, which is comparable with the 15 - $\mu$ s sample time.

The kinetic rate constants used for the theoretical curves in Fig. 3 were adjusted to produce a good agreement between theory and experiment, but most are reasonable based on other known spectroscopic facts. The $\mathrm{G} \rightarrow \mathrm{S}$ photon absorption rate $k_{\alpha}$ was set at $7.36 \times 10^{-17}$ $\mathrm{cm}^{2}$ based on the rhodamine extinction coefficient of $44300 \mathrm{~cm}^{-1} \mathrm{M}^{-1}$ (Molecular Probes, Inc.). The second photon absorption rate $k_{\beta}$ was set at $1 \times 10^{-18} \mathrm{~cm}^{2}$, nearly two orders of magnitude smaller than the first photon absorption rate. The $S \rightarrow G$ fluorescence decay rate $k_{\mathrm{f}}$ was set at $3.6 \times 10^{9} \mathrm{~s}^{-1}$, which is reasonable for a dye with a natural lifetime of several nanoseconds and a low quantum efficiency when bound to protein. The single-photon irreversible bleaching rate $k_{\mathrm{b}}$ was set at $5 \mathrm{~s}^{-1}$, which as expected is many orders of magnitude smaller than the rate of fluorescence. The only surprising value is that for the intersystem crossing rate $k_{\mathrm{i}}$, set at 6 $\times 10^{8} \mathrm{~s}^{-1}$. This is much faster than previously inferred [16], but the very large reversible bleaching component demands a high triplet-state population in our model.

\section{DISCUSSION}

The conventional view of photobleaching as it is practiced in FRAP is that it is both an irreversible and a rare event: A fluorophore goes through an average of thousands of cycles of excitation/deexcitation before it (or a neighbor) becomes bleached. If this view is correct, then bleaching by a pulse of light much shorter than the fluorescence lifetime should lead to virtually no bleaching at all, regardless of the bleaching intensity. In that 
case, a typical fluorophore will return to the ground state only after the bleaching pulse is terminated and thereby have only one tiny chance to become bleached.

We show here that (a) short-pulse bleaching is actually very efficient; (b) it is predominantly reversible at lower intensities; (c) it is predominantly irreversible at higher intensities; (d) both the reversible and the irreversible bleaching probabilities exhibit a nonlinear dependence on $I$, with the former slower than linear and the latter faster than linear; (e) the results can all be explained semiquantitatively by a simple three-state model that exhibits both saturation and two-photon effects and also accounts for the more familiar long pulse bleaching; and (f) the total bleaching exhibits a bleaching polarization anisotropy, making short-pulse photobleaching usable in PFRAP applications for fast rotational diffusion measurements.

The observation that different photobleaching mechanisms must operate for short- vs long-pulse bleaches is evident from the number of bleach photons involved. Our pulsed laser casts upon the sample in one short pulse $(0.5 \mathrm{~ns})$ typically one to three orders of magnitude fewer photons than delivered by a $\mathrm{CW}$ laser in a typical long pulse $(0.5 \mathrm{~ms})$ to achieve the same $(-50 \%)$ bleaching depth. Clearly, photobleaching is far more likely to be seen on short time scales (because of reversible bleaching) and more likely to occur with the higher pulsed laser intensities (because of two-photon processes).

The intensity of the bleaching light is huge: $10^{12}$ $\mathrm{W} / \mathrm{cm}^{2}$ at the center of the focused spot. But the heating due to absorption by the dye is only of the order $10^{-20} \mathrm{C}$, as can be calculated according to the method of Axelrod [17], which takes into account the dissipation of heat into the water. The main reason for the negligible heating is that each molecule becomes excited only, at most, a few times during the pulse; the ground state becomes rapidly depleted and the sample is effectively transparent during most of the duration of the 0.5 -ns pulse.

The model presented successfully predicts the main features of the intensity dependence of both the reversible and the irreversible bleaching components. But the details of the model are probably oversimplifications in several ways, including the following. (a) The standard single-photon long-pulse irreversible bleaching, depicted by the $k_{\mathrm{b}}$ route, probably consists of more than one pathway. Deoxygenation is known to suppress this route but it does not entirely eliminate it, implying some complexity to the process. One possible irreversible bleaching pathway involves energy transfer to dissolved oxygen, forming excited-singlet oxygen. This excited singlet then diffuses away to destroy other fluorophores in the ground state. (b) The two-photon pathway, assumed always to lead to irreversible bleaching, may be more complex. It is possible that a fluorophore excited to a high level by sequential photons may find its way back to the ground state without damage. (c) The model ignores the effects of bleaching and probe light polarization and restricted rotational diffusion of the fluorophores on the presumably "immobilized" sample. It is possible that some part of the fastest component of the reversible recovery may arise from fluorophore orientational motion. Because of all these complexities and possibly others, the kinetic rates needed in our simple model to fit the data should not be overinterpreted.

One of the most interesting future directions will be to look at the effect of deoxygenation on the depth and recovery time of subnanosecond bleaching. Particularly in view of the high rate of conversion to the triplet state conjectured in our kinetic model, oxygen in our samples may be quenching the triplet state and hastening the return to the ground state. If so, deoxygenation should have the effect of slowing the spontaneous postbleach recovery.

We have not examined polarization effects other than checking that the immediately postbleach bleaching anisotropy for a short pulse is high, in fact higher than seen for long-pulse bleaches on the same sample. Qualitatively, this means that those fluorophores with their $\mathrm{G} \rightarrow \mathrm{S}$ transition dipoles oriented parallel to the bleaching pulse polarization are the most likely to have been bleached. Although this may seem obvious for singlephoton bleaching, it is not necessarily true for two-photon bleaching, particularly if the transitions corresponding to the rates $k_{\alpha}$ and $k_{\beta}$ have dipole moments that are nonparallel to each other. The high initial bleaching anisotropy, combined with the easy bleachability, means that short pulse bleaching is feasible for use in a PFRAP system for studying biomolecular rotational motions with characteristic times over the entire range from submicroseconds to essentially infinite.

\section{ACKNOWLEDGMENTS}

We thank Drs. Andrea Stout and Robert Fulbright for help with curve-fitting software. This work was supported by NIH NS14565 and NSF DMB8805296.

\section{REFERENCES}

1. D. E. Wolf (1989) in D. L. Taylor and Y.-L. Wang. Fluorescence Microscopy of Living Cells in Culture. Part B. Methods in Cell Biology 30, Academic Press, New York, pp. 271-306.

2. M. Velez and D. Axelrod (1988) Biophys. J. 53, 575-591. 
3. T. M. Yoshida and B. G. Barisas (1986) Biophys. J. 50, 41-53. 4. P. Ravdin and D. Axclrod (1977) Anal. Biochem. 80, 585-592; and crratum, 83, 336.

5. R. Haugland (1983) in R. F. Stciner (Ed.), Excited States of Biopolymers, Plenum Press, Ncw York, pp. 29-58.

6. J. Lakowicz (1986) in D. L. Taylor, A. S. Waggoner, R. F. Murphy, F. Lanni, and R. R. Birge (Eds.), Applications of Fluorescence in the Biomedical Sciences, Alan R. Liss, Ncw York, pp. 29-67.

7. R. F. Fulbright and D. Axclrod (1993) J. Fluoresc. 3, 1-16.

8. D. Axclrod, J. Rubenstcin, and Y. Yuan (1992) Am. Chem. Soc. Polym. Prepr. 33, 755-756.

9. M. Velcz, K. F. Barald, and D. Axclrod (1990) J. Cell Biol. 110, 2049-2059.
10. B. Scalettar, P. Selvin, D. Axelrod, J. Hearst, and M. P. Klein (1990) Biochemistry 29, 4790-4798.

11. P. Sclvin, B. Scalettar, D. Axelrod, J. P. Langmorc, J. Hearst, and M. P. Klcin (1990) J. Mol. Biol. 214, 911-922.

12. F. Lanni and B. R. Ware (1981) Photochem. Photobiol. 34, 279281.

13. A. L. Stout and D. Axclrod (1994) Photochem. Photobiol., in press.

14. A. A. Gorman and M. A. J. Rodgers (1992) J. Photochem. Photobiol. B 14, 159-176.

15. O. H. Griffith, W. A. Houlc, K. F. Kongslie, and W. W. Sukow (1984) Ultramicroscopy 12, 299-307.

16. P. Johnson and P. Garland (1981) FEBS Lett. 132, 252-256.

17. D. Axelrod (1977) Biophys. J. 18, 129-131. 